\title{
Modelling of mass transfer coupling with crystallization kinetics in microscale
}

\author{
Yuanhui Ji ${ }^{a}$, Xiaoyan $\mathrm{Ji}^{\mathrm{b}}$, Chang Liu ${ }^{\mathrm{a}}$, Xin Feng ${ }^{\mathrm{a}}$, Xiaohua Lu ${ }^{\mathrm{a}, *}$ \\ a State Key Laboratory of Materials-Oriented Chemical Engineering, Nanjing University of Technology, Nanjing 210009, Jiangsu, P.R. China \\ ${ }^{\mathrm{b}}$ Division of Energy Engineering, Luleå University of Technology, SE-97187 Luleå, Sweden
}

\section{A R T I C L E I N F O}

\section{Article history:}

Received 26 May 2009

Received in revised form

29 October 2009

Accepted 30 December 2009

Available online 4 January 2010

Keywords:

Mass transfer

Microstructure

Microchannel

Crystallisation

Mathematical modelling

Microphase separation

\begin{abstract}
A B S T R A C T
Microstructure technologies have attracted interests in chemistry, chemical engineering, and biotechnology. To investigate the mass transfer of ions and crystallization of crystals in microscale and then to explain the formation mechanism of the porous structure materials, a microscale mathematical model for mass transfer processes coupling with local reactions is proposed in which the chemical potential gradient $\Delta \mu$ is used as the driving force to avoid the discontinuity of the kinetics equations in the micro-channels. Meanwhile, the dissolution kinetics of $\mathrm{KCl}$ at $298.15 \mathrm{~K}$ is measured to determine the dissolution rate constant $k_{d}$ and the average area of crystals $A c$. The investigation for the fractional crystallization process of carnallite shows that the calculated mixing time versus channel width agree with the Einstein diffusion equation, which validates that the model can be used to describe the ion diffusion very well. Meanwhile, to have an accurate $\Delta \mu$ of $\mathrm{KCl}$, in the channel width of or narrower than $2.0 \times 10^{-6} \mathrm{~m}$, it is enough to consider the diffusion only, while in the channel width of or wider than $2.0 \times 10^{-5} \mathrm{~m}$, diffusion should be coupled with reaction. The investigation also shows the vital of the consideration of the ionic activity coefficient for the investigated systems in micron scales. Moreover, the new formation mechanism of the porous structures in the inorganic material fabrication will be proposed from the process simulation for the synthesis of porous $\mathrm{KCl}$, which will provide a reference for the porous structure formation in the advanced inorganic material synthesis.
\end{abstract}

(c) 2010 Elsevier Ltd. All rights reserved.

\section{Introduction}

The development of microreaction technology is attractive for the multidisciplinary research and applications due to its advantages, such as increased yields, selectivity and product quality, improved heat transport and mass transport, offering the potential for process intensification, increased safety, decreased inputs and wastes, on-line reaction monitoring, etc. (Jensen, 1999; Hsing et al., 2000; Jensen, 2001; Chow, 2002; Kobayashi et al., 2004; Abdallah et al., 2006; Tagawa et al., 2007). Microchemical systems have been used in inorganic and organic material synthesis, nanoparticles synthesis, catalytic reactions, and photochemical and electrochemical reactions (Jensen, 1999; Stone and Kim, 2001; Jähnisch et al., 2004; Lin et al., 2004; Delamarche et al., 2005; Zhao et al., 2006; Mason et al., 2007; Tagawa et al., 2007).

The development of microchannel reaction technology offers tremendous multidisciplinary research opportunities across chemistry, materials, biology and electronics, as well as in the traditional chemical engineering subdisciplines of catalysis, transport phenomena, and reaction engineering (Jensen, 1999;

\footnotetext{
* Corresponding author. Tel./fax: +862583588063.

E-mail address: xhlu@njut.edu.cn (X. Lu).
}

Stone and Kim, 2001; Chow, 2002). Meanwhile, it also brings a lot of scientific and engineering challenges (Jensen, 1999; Stone and Kim, 2001). Whether the classical transport and reaction fundamentals are adequate to describe the transport and reaction behaviors in micro-channels and how the reaction affects the diffusion are two of these challenges.

Recently, computational fluid dynamics (CFD) has been used to describe the fluidic flow behaviors in microchannels (Schönfeld and Hardt, 2004; Yamaguchi et al., 2004) and to quantitatively estimate the mass transfer of microdevices (Abdallah et al., 2006; Schönfeld and Hardt, 2004; Yamaguchi et al., 2004; Salman et al., 2007). Abdallah et al. (2006) simulated the mass transfer of a catalytic multiphase microstructure film contactor with a fast hydrogenation of $\alpha$-methylstyrene and a $\mathrm{Pd} / \gamma$-alumina catalyst. However, the simulation results were inconsistent with the experimental results. This might be because that the concentration gradient is considered as the mass transfer driving force. Kashid et al. (2007) developed a CFD model to simulate the mass transfer with or without chemical reactions for the liquid-liquid slug flow capillary microreactor, and the diffusion driving force was also simplified to be the concentration gradient, which causes some errors in simulation.

Besides, in our previous work, porous structured $\mathrm{KCl}$ with a pore size of $10-50 \mu \mathrm{m}$ was produced from the fractional crystallization 
of carnallite $\left(\mathrm{KMgCl}_{3} \cdot 6 \mathrm{H}_{2} \mathrm{O}\right)$ (Liu et al., 2007). Moreover, Fick's second law was used to investigate the diffusion of the $\mathrm{K}^{+}, \mathrm{Mg}^{2+}$ and $\mathrm{Cl}^{-}$, and the ionic product of carnallite and $\mathrm{KCl}$ was analyzed to explain the formation mechanism of the porous $\mathrm{KCl}$ (Liu et al., 2007). However, in the fractional crystallization of carnallite, during the diffusion of ions, the dissolution or crystallization may take place at a certain position inside the carnallite crystal. In order to analyze the formation mechanism of the porous structure further and then to provide a reference for the porous structure formation, it is important to modify the theoretical model previously used.

In this work, a diffusion-reaction model will be proposed to analyze mass transfer and crystallization or dissolution in microscale systematically, in which the driving force of the diffusion and reaction will be represented with the potential gradient. The dissolution kinetics of $\mathrm{KCl}$ at $298.15 \mathrm{~K}$ will be investigated experimentally to determine the dissolution rate constant $k_{d}$ and the average area of crystals Ac. The diffusion of $\mathrm{K}^{+}, \mathrm{Mg}^{2+}, \mathrm{Cl}^{-}$and the crystallization kinetics of $\mathrm{KCl}$ in aqueous solutions will be studied in detail with the channel width in a range from $2.0 \times 10^{-7}$ to $0.2 \mathrm{~m}$, which covers the width of a microreactor (Chow, 2002; Jähnisch et al., 2004; Ehrfeld et al., 2000).

\section{Experimental section}

The dissolution kinetics of $\mathrm{KCl}$ at $298.15 \mathrm{~K}$ is investigated online using a chloride solid-state ion selective electrode (ISE) (Model pCl-1, China) and a calomel reference electrode. By sieving, the sizes of $\mathrm{KCl}$ crystals are in a range from $1.54 \times 10^{-4}$ to $3.00 \times 10^{-4} \mathrm{~m}$. The initial weight of $\mathrm{KCl}$ crystals is $3.950 \mathrm{~g}$. The concentrations of $\mathrm{Cl}^{-}$are measured by detecting the cell potential with a SCHOTT-GERÄTE pH-meter (Model CG0841, Germany) with a resolution of $\pm 0.1 \mathrm{mV}$.

The dissolution experiments are carried out in a double-walled crystallizer. The apparatus are described in Fig. 1. The pH-meter has a connecting interface to a personal computer and the potential data at a time interval of $1 \mathrm{~s}$ are automatically recorded. The constant stirring speed is $300 \mathrm{r} \mathrm{min}^{-1}$.

\section{Diffusion-reaction model}

The physical diffusion-reaction model can be schematically represented as shown in Fig. 2. In this model, ion diffusion and crystal crystallization/dissolution are considered while the fluid flow is neglected. The ion diffusion starts from the left boundary, and dissolution or crystallization may take place at a certain position inside the channel. The positive $\Delta \mu$ (chemical potential

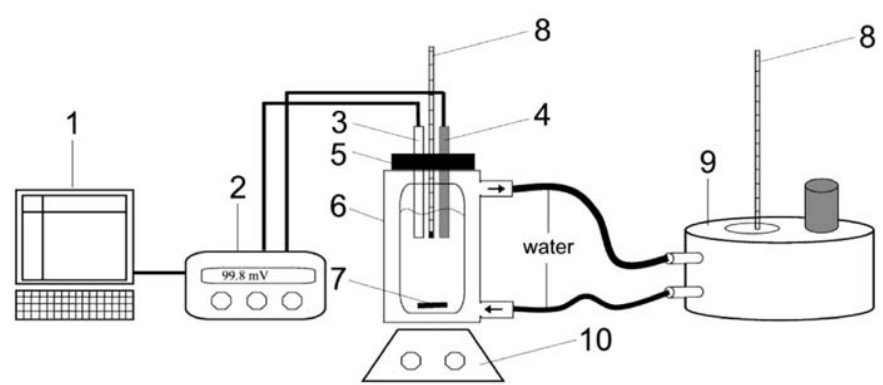

Fig. 1. Apparatus for measuring the dissolution of potassium chloride: $1 \mathrm{PC} ; 2 \mathrm{pH}$ meter; $3 \mathrm{Cl}^{-}$ISE; 4 calomel reference electrode; 5 rubber stopper; 6 double-walled crystallizer; 7 magnetic stir bar; 8 mercury thermometer; 9 thermostatic bath; 10 magnetic stirrer.

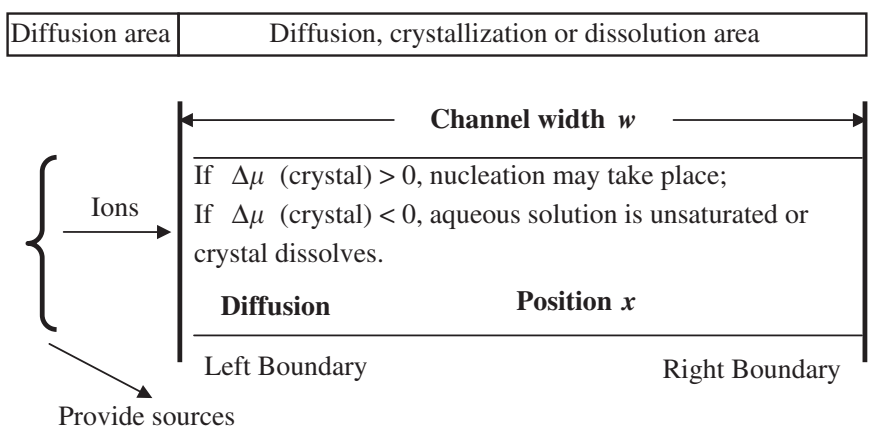

Fig. 2. Schematic representation of the diffusion-reaction model.

gradient) means that the aqueous solution is supersaturated and the crystallization may take place, the negative $\Delta \mu$ means that the aqueous solution is unsaturated or crystal dissolves, and the zero value of $\Delta \mu$ means that the aqueous solution is saturated and neither crystallization nor dissolution will happen.

The ion diffusion is governed by the convection-diffusion equation:

$\frac{\partial m}{\partial t}+u \cdot \nabla m=D \Delta m \pm r$

where $m$ is the molality of ions in aqueous solutions, $\mathrm{mol} \mathrm{kg}^{-1} ; t$ is time, $\mathrm{s} ; u$ is the flow velocity, $\mathrm{m} \mathrm{s}^{-1} ; D$ is the diffusivity, $\mathrm{m}^{2} \mathrm{~s}^{-1}$; and $r$ represents the source or consumption rate term depending on the kinetics of the reaction, $\mathrm{mol} \mathrm{kg}^{-1} \mathrm{~s}^{-1}$. In this work, the term $r$ is the coupling of the crystallization rate with the dissolution rate in the crystallization or dissolution process. That is

$r=A c(J-G)$

where $J$ and $G$ are the dissolution and crystallization rates $\left(\mathrm{mol} \mathrm{kg} \mathrm{k}^{-1} \mathrm{~m}^{-2} \mathrm{~s}^{-1}\right.$ ), respectively. The magnitude of $J$ and $G$ is dependent on chemical potential gradient. And $A c$ is the average area of the crystals, $\mathrm{m}^{2}$.

The dissolution rate is expressed as

$J=k_{d}(K s p-A s p)$

where $k_{d}$ is the dissolution rate constant, $\mathrm{mol} \mathrm{kg}^{-1} \mathrm{~m}^{-2} \mathrm{~s}^{-1} . K s p$ is the dimensionless solubility product constant, Asp is the dimensionless ionic product.

For the crystallization process, three kinds of crystal growth mechanisms have been proposed, i.e. spiral growth mechanism, two-dimensional nucleation growth mechanism, and adhesivetype mechanism (Sunagawa, 2005). In this work, the adhesivetype mechanism is adopted because the process investigated in this work is very fast and it is reasonable to assume that the nucleation and crystal growth occur simultaneously. Therefore, the crystallization rate is expressed as (Sunagawa, 2005)

$G=A\left(\frac{\Delta \mu}{R T}\right)=A \ln \left(\frac{A s p}{K s p}\right)$

where $A$ is a constant, $\mathrm{mol} \mathrm{kg}^{-1} \mathrm{~m}^{-2} \mathrm{~s}^{-1} ; \Delta \mu$ is the chemical potential difference at the solid-liquid interface (Cheng et al., 2006), $\mathrm{J} \mathrm{mol}^{-1} ; R$ is the universal gas constant, $\mathrm{J} \mathrm{mol}^{-1} \mathrm{~K}^{-1}$ and $T$ is the temperature, $\mathrm{K}$.

Therefore, for the static aqueous solution $(u=0)$, the diffusionreaction equation can be written as

$\frac{\partial m}{\partial t}=D \Delta m+A c \cdot k_{d}(K s p-A s p)-A c \cdot A \ln \left(\frac{A s p}{K s p}\right)$ 


\subsection{Fractional crystallization process of carnallite}

The fractional crystallization process of carnallite is selected as our study case since the carnallite produced is a material with micron to millimeter scales. In the fractional crystallization process of carnallite, the carnallite is dissolved, and $\mathrm{K}^{+}, \mathrm{Mg}^{2+}$ and $\mathrm{Cl}^{-}$begin to diffuse from the solid-liquid interface. Since the solubility of magnesium chloride is very large and the crystal magnesium chloride will never appear under the investigated conditions, the following equations can be obtained:

$\frac{\partial m_{K^{+}}}{\partial t}=D_{\mathrm{KCl}} \frac{\partial^{2} m_{K^{+}}}{\partial x^{2}}+A c k_{d}\left(K s p_{\mathrm{KCl}}-A s p_{\mathrm{KCl}}\right)-A c A \ln \left(\frac{A s p_{\mathrm{KCl}}}{K s p_{\mathrm{KCl}}}\right)$

$\frac{\partial m_{\mathrm{Mg}^{2+}}}{\partial t}=D_{\mathrm{MgCl}_{2}} \cdot \frac{\partial^{2} m_{\mathrm{Mg}^{2+}}}{\partial x^{2}}$

$\frac{\partial m_{\mathrm{Cl}^{-}}}{\partial t}=\frac{\partial m_{\mathrm{K}^{+}}}{\partial t}+2 \cdot \frac{\partial m_{\mathrm{Mg}^{2+}}}{\partial t}$

In Eq. (6)

$\operatorname{Asp}_{K C l}=\frac{m_{K^{+}}}{m^{\theta}} \cdot \frac{m_{C l}}{m^{\theta}} \cdot \gamma_{ \pm K C l}^{2}$

$K s p_{K C l}=\frac{m_{s, K^{+}}}{m^{\theta}} \cdot \frac{m_{C l^{-}}}{m^{\theta}} \cdot \gamma_{ \pm s, K C l}^{2}$

where $\gamma_{ \pm \mathrm{KCl}}$ is the mean ionic activity coefficient of $\mathrm{KCl}$ in the aqueous solution, and it is calculated with Lu-Maurer (1993) model. $m_{\mathrm{s}, \mathrm{K}}^{+}$is calculated on the basis of solid-liquid equilibrium in the aqueous $\mathrm{K}^{+}-\mathrm{Mg}^{2+}-\mathrm{Cl}^{-}$solution at a certain concentration of $\mathrm{Mg}^{2+} . x$ is the position inside a channel with a certain width $w$.

The driving force $\Delta \mu$ (difference of chemical potential or chemical potential gradient) of the crystallization or dissolution of $\mathrm{KCl}$ is calculated by the following equation:

$\Delta \mu_{\mathrm{KCl}}=R T \ln \left(\frac{A s p_{\mathrm{KCl}}}{K s p_{\mathrm{KCl}}}\right)$

\subsection{Parameter determination}

$k_{d}, D_{\mathrm{KCl}}, D_{\mathrm{MgCl}_{2}}, A c$ and $A$ are parameters in the proposed model for the investigated process. The areas of crystals at different time At are calculated by the method in Ji et al. (2001) according to the dissolution kinetics data. In the calculation of $A t$, the initial diameters of crystals are assumed to satisfy the normal distribution. Ac is obtained as an average area value. $k_{d}$ is determined with the modified statistical rate theory (SRT) (Dejmek and Ward, 1998; Ji et al., 2001) from the dissolution kinetics data that are measured in this work, which will be described in detail in the later part of this paper. The modified SRT (Dejmek and Ward, 1998; Ji et al., 2001) can be used to describe the instantaneous rate of molecular transport across the interface between the solid and liquid phases. The rate expression is in terms of (a) the chemical potential of the molecules in the solid; (b) the chemical potential of the molecules in the liquid solution at the interface with the solid phase and (c) the equilibrium exchange rate between the phases that would exist if the isolated system is allowed to evolve into equilibrium and both phases are present. For the modelling of the fractional crystallization process of carnallite, $D_{\mathrm{KCl}}$ and $D_{\mathrm{MgCl}_{2}}$ are assumed to be constants, and they are equal to the values in dilute solutions (Zaytsev and Aseyev, 1992).

$D_{\mathrm{KCl}}=1.856 \times 10^{-9}\left(\mathrm{~m}^{2} \mathrm{~s}^{-1}\right)$

$D_{\mathrm{MgCl}_{2}}=1.25 \times 10^{-9}\left(\mathrm{~m}^{2} \mathrm{~s}^{-1}\right)$
The parameter $A$ represents the Arrhenius relationship of the crystal growth rate coefficient related with the temperature and hydrodynamic state in solutions (Cheng et al., 2006). The value of $A$ for crystallization of $\mathrm{KCl}$ is considered to be similar to that of $\mathrm{K}_{2} \mathrm{SO}_{4}$ which is $0.4371 \mathrm{~mol} \mathrm{~kg}^{-1} \mathrm{~m}^{-2} \mathrm{~s}^{-1}$ at $298.15 \mathrm{~K}$.

\subsection{Discretization, initial and boundary conditions}

Galerkin method is used for spatial discretization of the governing equations. The details of the discretization method have been described by Skeel and Berzins (1990).

The ion diffusion in three aqueous solutions is investigated under the initial conditions listed in Table 1.

For the modelling of the fractional crystallization process of carnallite, at left boundary, $m_{\mathrm{K}^{+}}=3.1406 \mathrm{~mol} \mathrm{~kg}^{-1}, m_{\mathrm{Mg}^{2+}}=$ $3.1406 \mathrm{~mol} \mathrm{~kg}^{-1}$, and the molality of $\mathrm{Cl}^{-}$is determined according to the neutrality of charges, and the right boundary conditions are the same as the initial conditions in the bulk phase.

\section{Results and discussions}

\subsection{Experimental results}

To determine the dissolution rate constant for the diffusionreaction model, the dissolution kinetics of $\mathrm{KCl}$ at $298.15 \mathrm{~K}$ is investigated experimentally. The experimental results are shown in Fig. 3. As shown in Fig. 3, the molality of chloride ion increases sharply with time at the beginning of the dissolution process, and then increases slowly and becomes a constant value, which means the dissolution has finished.

Table 1

Initial conditions for the diffusion-reaction model.

\begin{tabular}{llll}
\hline Solutions & $\begin{array}{l}\mathrm{K}^{+} \text {concentration } \\
\left(\mathrm{mol} \mathrm{kg}^{-1}\right)\end{array}$ & $\begin{array}{l}\mathrm{Mg}^{2+} \text { concentration } \\
\left(\mathrm{mol} \mathrm{kg}^{-1}\right)\end{array}$ & $\begin{array}{l}\mathrm{Cl}^{-} \text {concentration } \\
\left(\mathrm{mol} \mathrm{kg}^{-1}\right)\end{array}$ \\
\hline $\begin{array}{c}\mathrm{KCl} \text { aqueous } \\
\text { solution } \\
\mathrm{MgCl}_{2} \text { aqueous } \\
\text { solution }\end{array}$ & 3.930 & 0 & 3.930 \\
Distilled water & 0 & 0 & 6.302 \\
\hline
\end{tabular}

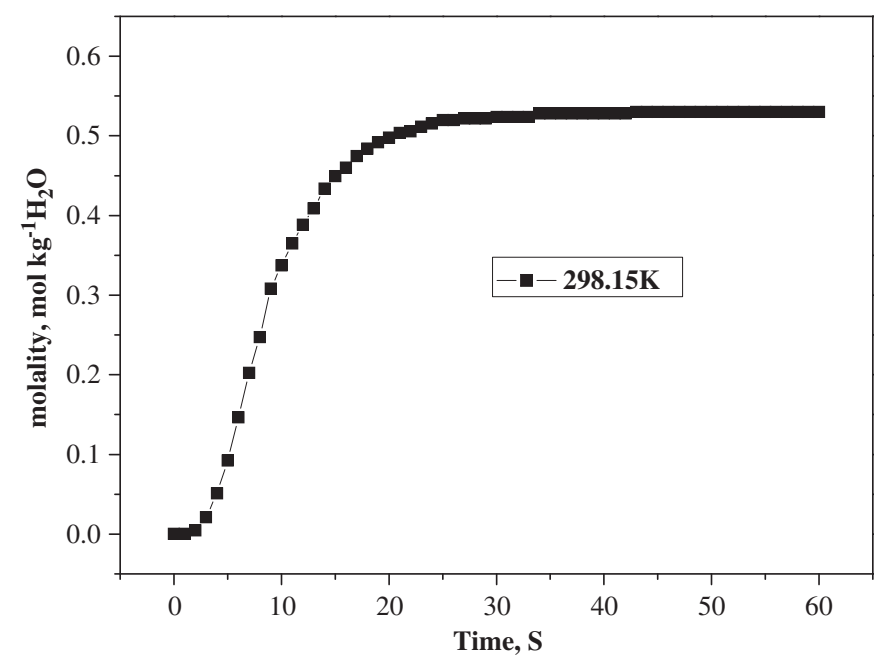

Fig. 3. Molality of chloride ions with time during the dissolution process of $\mathrm{KCl}$ crystals at $298.15 \mathrm{~K}$. 
The changing of areas of crystals with time is considered in this work. According to the dissolution kinetics data, the areas of crystals at different time At are calculated by the method in Ji et al. (2001) in which the initial diameters of crystals are assumed to satisfy the normal distribution. Ac is obtained as an average value for the areas of the crystals at different time, with the value of $3.639 \times 10^{-4} \mathrm{~m}^{2} . k_{d}$ is obtained with the statistical rate theory (SRT) (Dejmek and Ward, 1998; Ji et al., 2001) with the value of $6.000 \times 10^{-4} \mathrm{~m} \mathrm{~s}^{-1}$.

\subsection{Model results}

To investigate the effects of reaction term $r$ in Eq. (6) and activity coefficient in Eqs. (9)-(11) on diffusion, the model in three cases is performed, i.e.

case 1: considering diffusion but neglecting reaction, i.e. $r=0$ in Eq. (6);

case 2: considering diffusion coupling with reaction; and case 3: considering diffusion coupling with reaction but activity coefficient is assumed to be unity in the calculation of Asp and Ksp.

\subsubsection{Mixing time in different aqueous solutions}

Mixing time can be obtained from the value of $\Delta \mu$ with time. When $\Delta \mu$ keeps a constant throughout the whole channel, the mixing process is completed. The calculated mixing time versus channel width is shown in Fig. 4 where the double logarithmic coordinate axes are used to describe the large range of time and channel width.

In Stokes flow characteristic in microchannels, mixing time $(t)$ is dictated by thermal diffusion described approximately by the Einstein diffusion equation (Chow, 2002):

$t=w^{2} /(2 D)$

where $w$ is the channel width and $D$ is the molecular diffusion coefficient.

As shown in Fig. 4(a), the logarithm of the mixing time calculated with the model in case 1 is proportional to the logarithm of the channel width in the solutions of aqueous $\mathrm{KCl}$, aqueous $\mathrm{MgCl}_{2}$ and distilled water, which follows the Einstein diffusion equation (Eq. (14)). This observation validates that the model can be used to describe the diffusion of ions very well. The calculated results also reveal that the mixing time can be highly reduced by shortening the channel width.

With the model in case 2, as shown in Fig. 4(b), in aqueous $\mathrm{KCl}$ solutions, an almost linear relationship between the logarithms of the mixing time and the logarithms of channel width is obtained. However, in the solution of aqueous $\mathrm{MgCl}_{2}$ and distilled water, the dual logarithm relationship of the mixing time versus channel width is nonlinear, especially for the wide channel, and the mixing time in these two solutions is obvious different for the channel width of $2.0 \times 10^{-3} \mathrm{~m}$.

As shown in Fig. 4(c), with the model in case 3, the dual logarithm relationship of the mixing time versus channel width is linear for the channel width of and narrower than $2.0 \times 10^{-4} \mathrm{~m}$. Fig. 4(c) also shows nearly the same mixing time in aqueous $\mathrm{MgCl}_{2}$ solutions and distilled water. These observations validates that ion diffusion can be described with the model in the cases from 1 to 3 . These observations agree with those from the view of Jensen (1999) that the mixing occurs primarily by diffusion for the small widths in microreactor channels. a

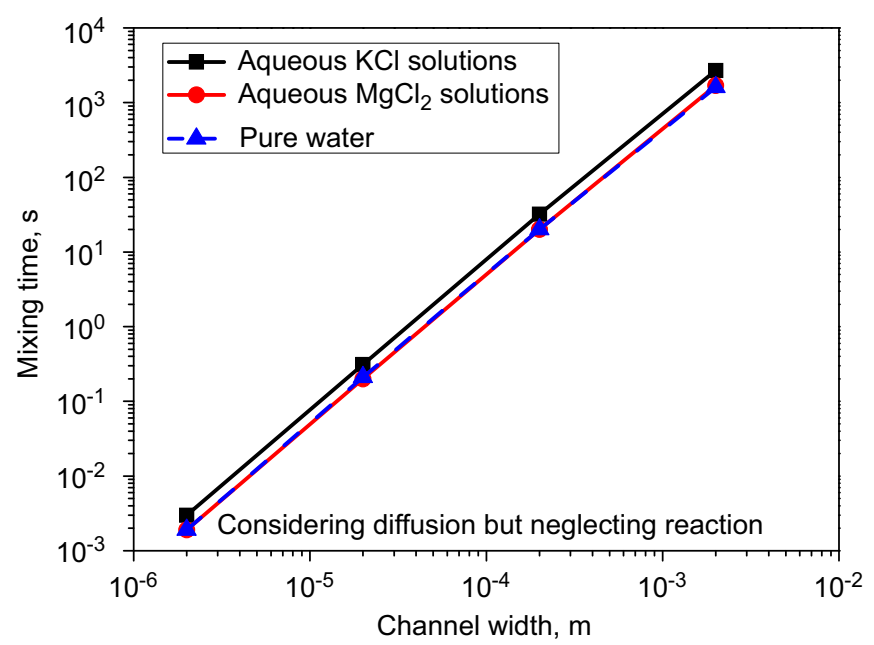

b

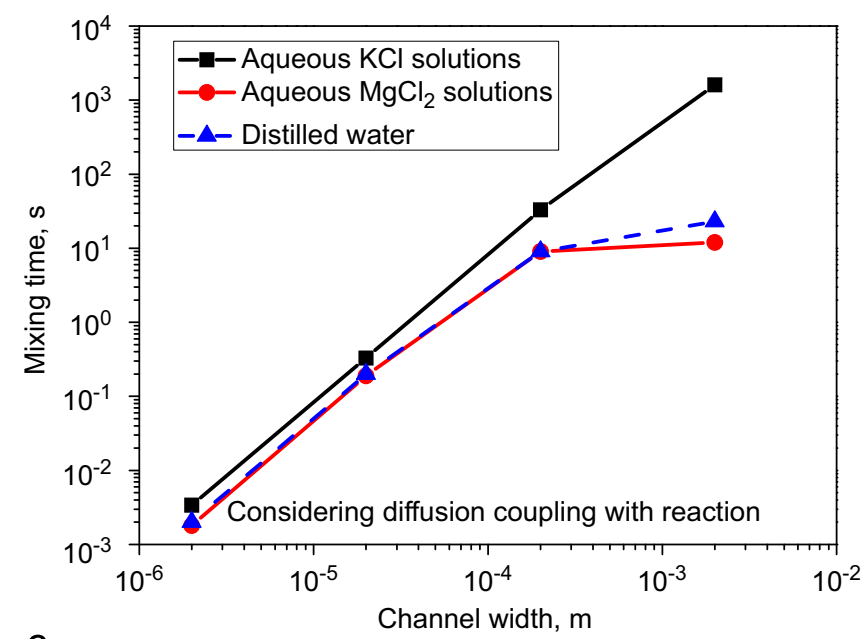

C

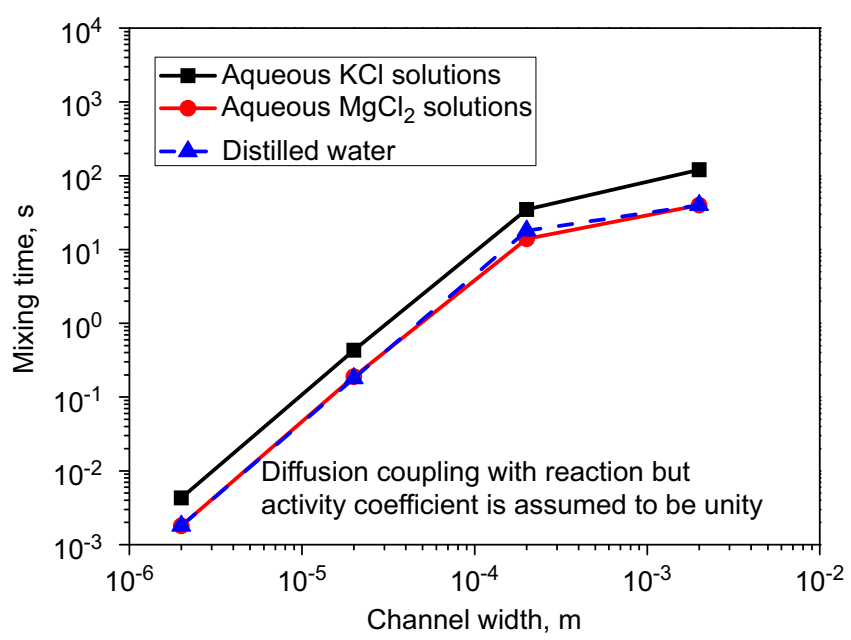

Fig. 4. Mixing time at different channel widths calculated with the model in different cases: (a) in case 1, diffusion neglecting reaction; (b) in case 2, diffusion coupling with reaction; and (c) in case 3 , diffusion coupling with reaction but the value of activity coefficient is unity.

\subsubsection{Diffusion and reaction behaviors inside the channel}

The value of $\Delta \mu$ of $\mathrm{KCl}$ in distilled water calculated by assuming the activity coefficient to be unity is always less than zero, which disagrees with the experimental observations (Liu et al., 2007). 
Therefore, in the following investigation, the activity coefficient is always calculated with Lu-Maurer (1993) model.

$\Delta \mu$ of $\mathrm{KCl}$ inside a channel under the initial condition of distilled water is investigated in detail. In calculation, the channel widths are set to be $2 \times 10^{-6}, 2 \times 10^{-5}, 2 \times 10^{-4}, 2 \times 10^{-3}, 0.02$ and $0.2 \mathrm{~m}$, respectively, which covers the width of a microreactor (Chow, 2002; Jähnisch et al., 2004; Ehrfeld et al., 2000). $\Delta \mu$ of $\mathrm{KCl}$ at different position inside the channel is calculated. Fig. 5 (A1, A2) shows the results at $0.001,0.1$ and $10 \mathrm{~s}$ calculated with the model in case 1 and Fig. 6 (B1, B2, B3) shows those at 0.001, 1 and 1200 s calculated with the model in case 2 .
As shown in Fig. 5 (A1, A2, (a-c)), fluctuations may appear. At $0.001 \mathrm{~s}$, the fluctuations appear in the channel widths of $2.0 \times 10^{-5}$ and $2.0 \times 10^{-4} \mathrm{~m}$. At $0.1 \mathrm{~s}$, the fluctuations appear in the channel widths of $2.0 \times 10^{-4}$ and $2.0 \times 10^{-3} \mathrm{~m}$. At $10 \mathrm{~s}$, the fluctuations occur in the channel widths of $2.0 \times 10^{-3}$ and $2.0 \times 10^{-2} \mathrm{~m}$. For the channel width of $2.0 \times 10^{-6} \mathrm{~m}$, no fluctuation appears at any time.

As shown in Fig. 6 (B1), at $0.001 \mathrm{~s}$, fluctuation appears only in the channel widths of $2.0 \times 10^{-5}$ and $2.0 \times 10^{-4} \mathrm{~m}$. At 1 and $1200 \mathrm{~s}$, no obvious fluctuation appears in the channel widths of $2.0 \times 10^{-6}$, $2.0 \times 10^{-5}, 2.0 \times 10^{-4}$ and $2.0 \times 10^{-3} \mathrm{~m}$. From Fig. 6 (B2, B3), we can observe obvious fluctuations appear at different time in the

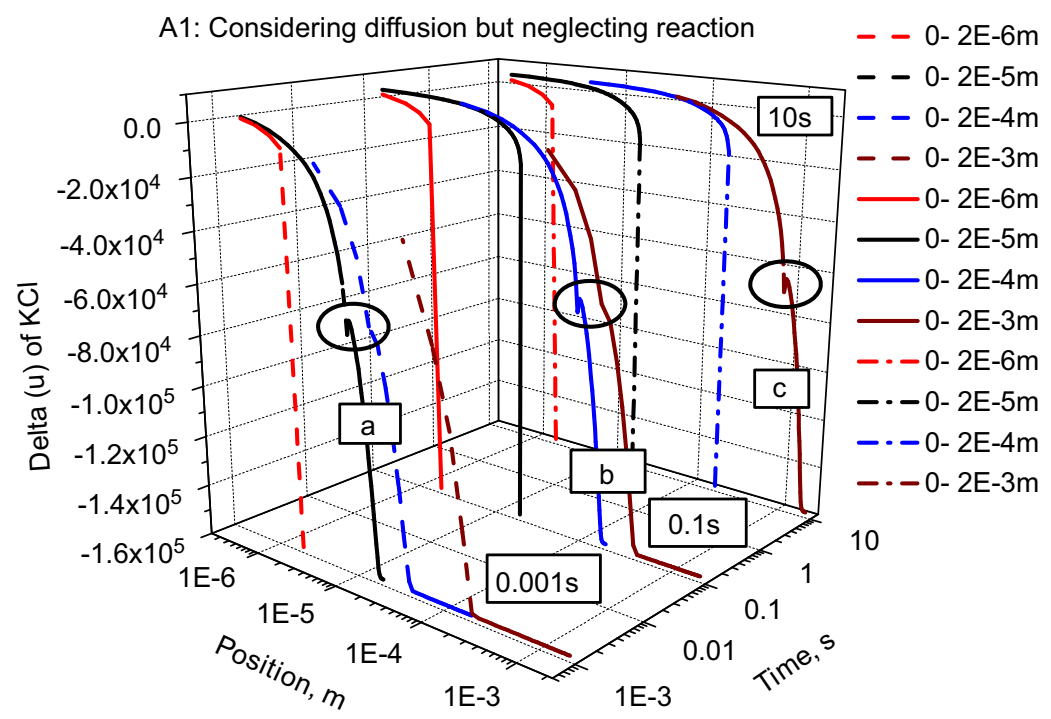

A2: Considering diffusion but neglecting reaction

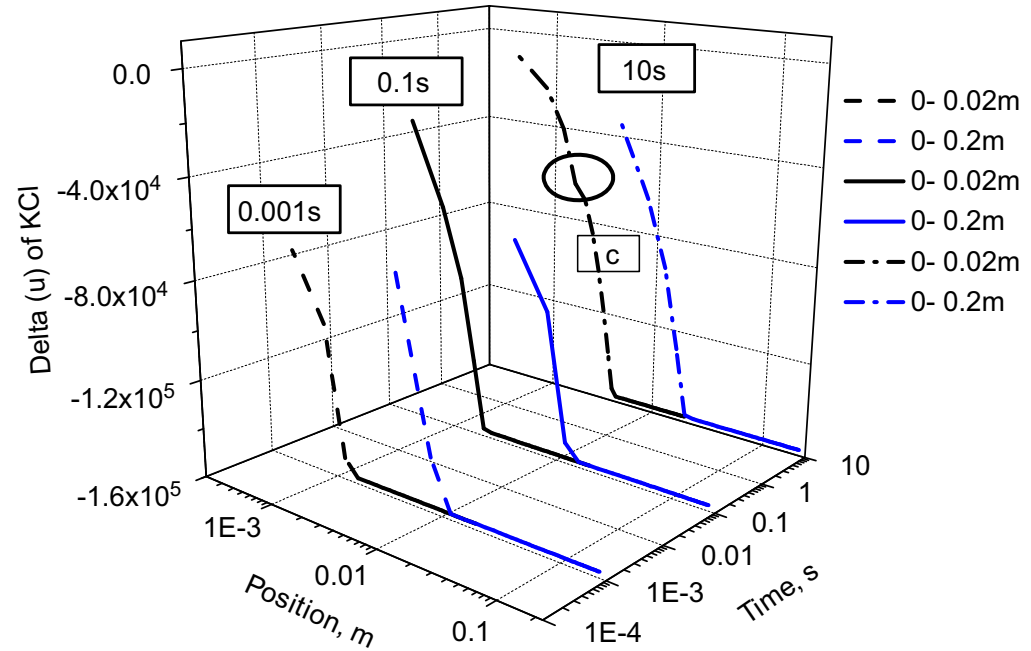

a

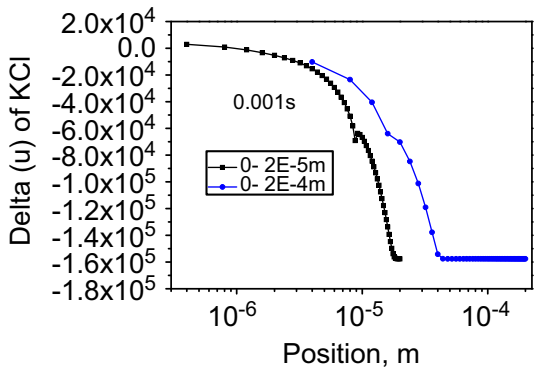

b

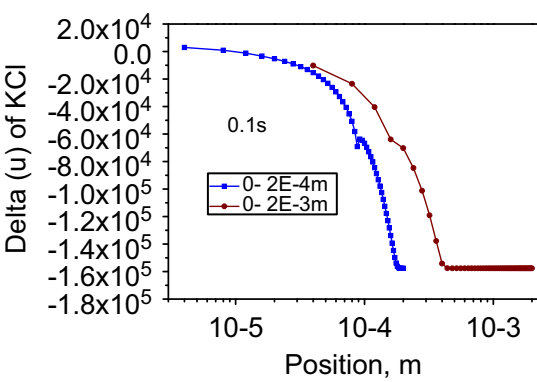

C

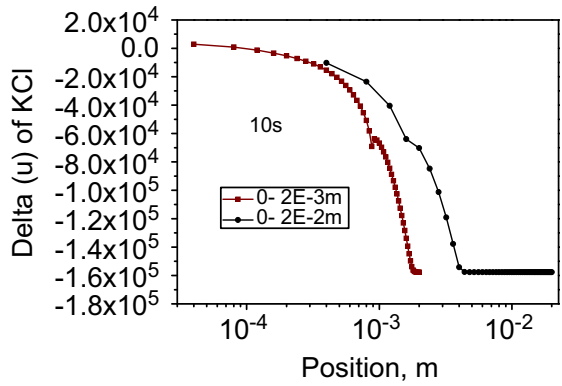

Fig. 5. $\Delta \mu$ of $\mathrm{KCl}$ in distilled water calculated with the model considering diffusion but neglecting reaction (model in case 1 ). 


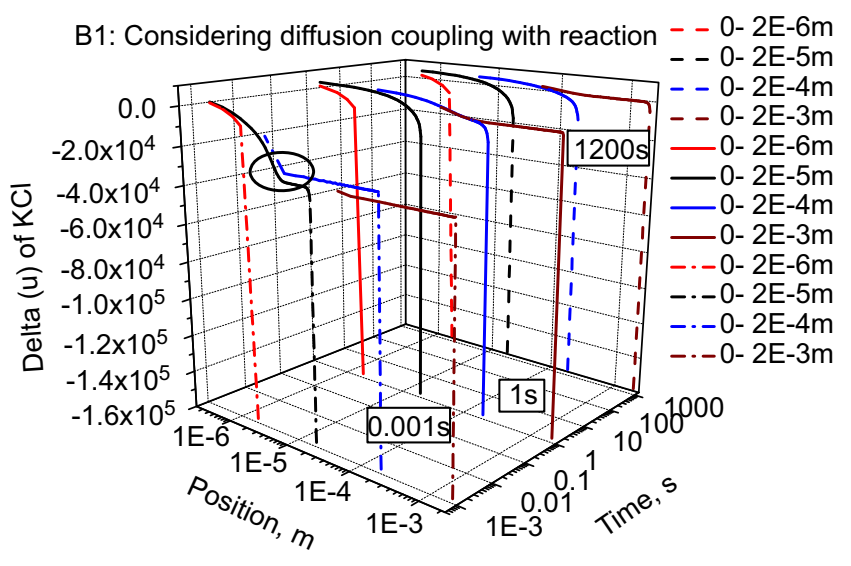

B2: Considering diffusion coupling with reaction

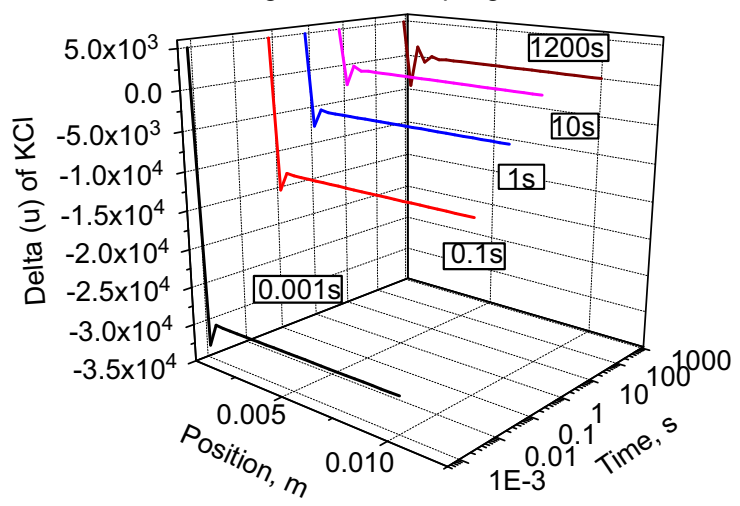

B3: Considering diffusion coupling with reaction: $0-0.2 \mathrm{~m}$
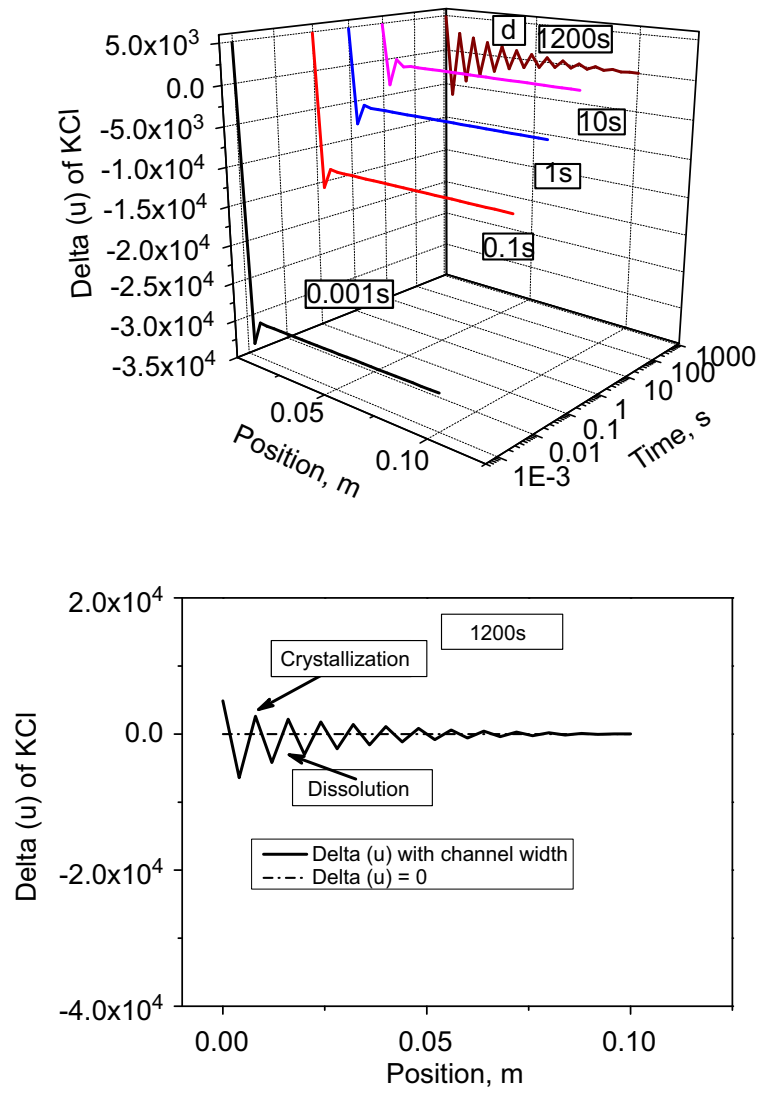

Fig. 6. $\Delta \mu$ of $\mathrm{KCl}$ in distilled water calculated with the model considering diffusion coupling with reaction (model in case 2).

channel widths of 0.02 and $0.2 \mathrm{~m}$. The fluctuations of the $\Delta \mu$ of $\mathrm{KCl}$ around $\Delta \mu=0$ in Fig. 6 (B2, B3) give an explanation on how the porous structures form after the carnallite dissolving in distilled water, i.e. when $\Delta \mu$ is positive, the aqueous solution is supersaturated and the crystallization may occur; when $\Delta \mu$ is negative, the aqueous solution is unsaturated or the crystals which have been formed may dissolve again. As shown in Fig. 6 (B3(d)), the magnitude of $\Delta \mu$ for the dissolution of $\mathrm{KCl}$ is larger than that for the nucleation and the differences between these two driving forces near the carnallite boundary lead to the porous crystal structures.

From the results shown in Figs. 5 and 6, we can observe that in the channel width of $2.0 \times 10^{-6} \mathrm{~m}$ the $\Delta \mu$ of $\mathrm{KCl}$ obtained in the cases of diffusion with and without reactions is the same, and the same for the channel width of $2.0 \times 10^{-7} \mathrm{~m}$. While in the channel width of or wider than $2.0 \times 10^{-5} \mathrm{~m}$, the $\Delta \mu$ of $\mathrm{KCl}$ obtained in the case of diffusion neglecting reaction (case 1 ) is different from that obtained in the case of diffusion coupling with reaction (case 2). Therefore, we may conclude that in the channel width of or narrower than $2.0 \times 10^{-6} \mathrm{~m}$, the contribution of reaction to the $\Delta \mu$ of $\mathrm{KCl}$ can be neglected, while in the channel widths of or wider than $2.0 \times 10^{-5} \mathrm{~m}$, both diffusion and reaction have to be considered in order to obtain a reliable of $\mathrm{KCl}$.

\section{Conclusions}

In this work, a microscale mathematical model for local reactions and mass transfer processes is proposed to investigate the mass transfer of ions and nucleation of crystals in microscale, and the chemical potential gradient instead of concentration gradient is used as the driving force to avoid the discontinuity of the kinetics equations in the micro-channels. Porous structured $\mathrm{KCl}$ with a pore size of $10-50 \mu \mathrm{m}$ is produced and the dissolution kinetics of $\mathrm{KCl}$ at $298.15 \mathrm{~K}$ is investigated experimentally to determine the dissolution rate constant $k_{d}$ and the average area of crystals $\mathrm{Ac}$. For the aqueous $\mathrm{K}-\mathrm{Mg}-\mathrm{Cl}$ systems, the dual logarithm relationship of the calculated mixing time versus channel width is linear, which agrees with the Einstein diffusion equation. This validates that the model can be used to describe the diffusion of ions very well. Meanwhile, in the channel width of or narrower than $2.0 \times 10^{-6} \mathrm{~m}$, the contribution of reaction to the $\Delta \mu$ of $\mathrm{KCl}$ can be neglected, while in the channel width of or wider than $2.0 \times 10^{-5} \mathrm{~m}$, both diffusion and reaction have to be considered. It is also shown the vital of the mean ionic activity coefficient for the investigated systems in micron scales. Moreover, the calculated results of the proposed model reveal the formation mechanism of the porous structures in the inorganic material fabrication. This will provide a reference for the regular and ordered porous structure formation and microphase separation mechanisms in the advanced inorganic material synthesis.

\section{Acknowledgments}

This work was supported by the Chinese National Key Technology Research and Development Program (Grant nos. 2006BAB09B02, 2006AA03Z455 and 2006BAE03B7-2), the Chinese National Science Foundation for Outstanding Young Scholars 
(no. 20428606), the Natural Science Foundation of the Jiangsu Higher Education Institutions of China (08KJB530003), the National Natural Science Foundation of China (Grant nos. 20676062, 20731160614, 20736002, B061101, B060105, 20706029 and 20706028), NSFC-RGC (no. 20731160614), the Key Science Foundation of Jiangsu Province, China (BK 2004215) and Program for Changjiang Scholars and Innovative Research Team in University (no. IRT0732).

\section{References}

Abdallah, R., Magnico, P., Fumey, B., de Bellefon, C., 2006. CFD and kinetic methods for mass transfer determination in a mesh microreactor. A.I.Ch.E. Journal 52, 2230-2237.

Chow, A.W., 2002. Lab-on-a-chip: opportunities for chemical engineering. A.I.Ch.E. Journal 48, 1590-1595.

Cheng, F.Q., Bai, Y., Liu, C., Lu, X.H., Dong, C., 2006. Thermodynamic analysis of temperature dependence of the crystal growth rate of potassium sulfate. Industrial and Engineering Chemistry Research 45, 6266-6271.

Delamarche, E., Juncker, D., Schmid, H., 2005. Microfluidics for processing surfaces and miniaturizing biological assays. Advanced Materials 17, 2911-2933.

Dejmek, M., Ward, C.A., 1998. A statistical rate theory study of interface concentration during crystal growth or dissolution. The Journal of Chemical Physics 108, 8698-8704.

Ehrfeld, W., Hessel, V., Löwe, H., 2000. Microreactors: New Technology for Modern Chemistry. Wiley-VCH, Weinheim.

Hsing, I.M., Srinivasan, R., Harold, M.P., Jensen, K.F., Schmidt, M.A., 2000. Simulation of micromachined chemical reactors for heterogeneous partial oxidation reactions. Chemical Engineering Science 55, 3-13.

Jensen, K.F., 1999. Microchemical systems: status, challenges, and opportunities. A.I.Ch.E. Journal 45, 2051-2054

Jensen, K.F., 2001. Microreaction engineering-is small better? Chemical Engineering Science 56, 293-303.

Jähnisch, K., Hessel, V., Löwe, H., Baerns, M., 2004. Chemistry in microstructured reactors. Angewandte Chemie International Edition 43, 406-446.

Ji, X.Y., Chen, D.L., Wei, T., Lu, X.H., Wang, Y.R., Shi, J., 2001. Determination of dissolution kinetics of $\mathrm{K}_{2} \mathrm{SO}_{4}$ crystal with ion selective electrode. Chemical Engineering Science 56, 7017-7024.
Kobayashi, J., Mori, Y., Okamoto, K., Akiyama, R., Ueno, M., Kitamori, T., Kobayashi, S., 2004. A microfluidic device for conducting gas-liquid-solid hydrogenation reactions. Science 304, 1305-1308.

Kashid, M.N., Agar, D.W., Turek, S., 2007. CFD modelling of mass transfer with and without chemical reaction in the liquid-liquid slug flow microreactor. Chemical Engineering Science 62, 5102-5109.

Lin, X.Z., Terepka, A.D., Yang, H., 2004. Synthesis of silver nanoparticles in a continuous flow tubular microreactor. Nano Letters 4, 2227-2232.

Liu, C., Ji, Y.H., Bai, Y., Cheng, F.Q., Lu, X.H., 2007. Formation of porous crystals by coupling of dissolution and nucleation process in fractional crystallization. Fluid Phase Equilibria 261, 300-305.

Lu, X.H., Maurer, G., 1993. Model for mixed describing electrolyte activity coefficients in aqueous solutions. A.I.Ch.E. Journal 39, 1527-1538.

Mason, B.P., Price, K.E., Steinbacher, J.L., Bogdan, A.R., McQuade, D.T., 2007. Greener approaches to organic synthesis using microreactor technology. Chemical Reviews 107, 2300-2318.

Sunagawa, I., 2005. Crystals-Growth, Morphology and Perfection. Cambridge University Press, Cambridge.

Stone, H.A., Kim, S., 2001. Microfluidics: basic issues, applications, and challenges. A.I.Ch.E. Journal 47, 1250-1254.

Schönfeld, F., Hardt, S., 2004. Simulation of helical flows in microchannels. A.I.Ch.E. Journal 50, 771-778.

Salman, W., Gavriilidis, A., Angeli, P., 2007. Axial mass transfer in taylor flow through circular microchannels. A.I.Ch.E. Journal 53, 1413-1428.

Skeel, R.D., Berzins, M., 1990. A method for the spatial discretization of parabolic equations in one space variable. SIAM Journal on Scientific and Statistical Computing 11, 1-32.

Tagawa, T., Aljbour, S., Matoup, M., Yamada, H., 2007. Micro-channel reactor with guideline structure for oganic-aqueous binary system. Chemical Engineering Science 62, 5123-5126.

Yamaguchi, Y., Takagi, F., Yamashita, K., Nakamura, H., Maeda, H., Sotowa, K., Kusakabe, K., Yamasaki, Y., Morooka, S., 2004. 3-D simulation and visualization of laminar flow in a microchannel with hair-pin curves. A.I.Ch.E. Journal 50, 1530-1535.

Zhao, Y.C., Chen, G.W., Yuan, Q., 2006. Liquid-liquid two-phase flow patterns in a rectangular microchannel. A.I.Ch.E. Journal 52, 4052-4060.

Zaytsev, I.D., Aseyev, G.G., 1992. Properties of Aqueous Solutions of Electrolytes. CRC Press, Boca Raton, FL. 